\title{
Masked nocturnal hypertension-a novel marker of risk in type 2 diabetes
}

\author{
M. Wijkman • T. Länne • J. Engvall • T. Lindström • \\ C. J. Östgren • F. H. Nystrom
}

Received: 6 February 2009 /Accepted: 30 March 2009/Published online: 25 April 2009

(C) Springer-Verlag 2009

\begin{abstract}
Aims/hypothesis This study was designed to evaluate the prevalence of masked nocturnal hypertension (MNHT) and its impact on arterial stiffness and central blood pressure in patients with type 2 diabetes.

Methods Middle-aged patients $(n=414)$ with type 2 diabetes underwent clinic and ambulatory BP measurements and applanation tonometry.

Results MNHT (clinic $\mathrm{BP}<130 / 80 \mathrm{mmHg}$ and night-time $\mathrm{BP} \geq 120 / 70 \mathrm{mmHg})$ was found in $7.2 \%$ of patients $(n=30)$. Compared with patients with both clinical and nocturnal normotension $(n=70)$, patients with MNHT had higher aortic pulse wave velocity (PWV) $(10.2 \pm 1.8 \mathrm{~m} / \mathrm{s}$ vs $9.4 \pm 1.7 \mathrm{~m} / \mathrm{s}$; $p=0.03)$ and higher central BP $(117.6 \pm 13.9 / 74.0 \pm 9.1 \mathrm{mmHg}$ vs $110.4 \pm 16.4 / 69.7 \pm 9.6 \mathrm{mmHg}, p=0.04)$. In patients with clinical normotension, night-time systolic BP correlated significantly with PWV.

Conclusions/interpretation Thirty per cent of patients with clinical normotension had nocturnal hypertension. This was accompanied by increased arterial stiffness and higher central BP. We conclude that in clinically normotensive patients with type 2 diabetes, ambulatory BP measurement may help clinicians to identify patients with increased cardiovascular risk.
\end{abstract}

M. Wijkman $(\bowtie) \cdot$ T. Länne $\cdot$ J. Engvall $\cdot$ T. Lindström •

C. J. Östgren $\cdot$ F. H. Nystrom

Department of Medical and Health Sciences,

Faculty of Health Sciences, Linköping University,

581 83, Linköping, Sweden

e-mail: magnus.wijkman@liu.se

e-mail: magnus.wijkman@lio.se

M. Wijkman • T. Lindström • C. J. Östgren · F. H. Nystrom

Diabetes Research Centre, Faculty of Health Sciences,

Linköping University,

Linköping, Sweden
Keywords Ambulatory blood pressure · Arterial stiffness · Hypertension
Abbreviations
BP Blood pressure
CARDIPP Cardiovascular Risk Factors in Patients with
LVMI Left ventricular mass index
MNHT Masked nocturnal hypertension
PWV Pulse wave velocity

\section{Introduction}

In type 2 diabetes, masked hypertension-defined as normal blood pressure when measured in the clinic but elevated BP when measured out of the clinic during the awake part of the day-is associated with an increased prevalence of retinopathy, nephropathy and coronary heart disease [1] and with target organ damage [2-4]. However, it is not known whether masked nocturnal hypertension (MNHT) - defined as normal BP when measured in the clinic but elevated BP when measured away from the clinic during the night - is a more useful tool for the identification of patients at high risk than masked hypertension. Arterial stiffness, as evaluated by aortic pulse wave velocity (PWV) measurements, is known to be increased in patients with type 2 diabetes and has been shown to predict mortality in these patients [5], but the associations between arterial stiffness and masked hypertension defined by either day- or night-time out-of-clinic BP measurements have not yet been described in type 2 diabetes. Furthermore, the prevalence of elevated night-time BP in patients with type 2 diabetes who have well-controlled clinic-measured BP is not known. Therefore, we analysed clinic, central and 
ambulatory BP measurements and aortic PWV measurements in 414 patients with type 2 diabetes with or without previously diagnosed hypertension. We also measured non-invasively in all patients the central aortic BP, an independent predictor of cardiovascular morbidity in the general population [6] and central augmentation index, a surrogate marker of arterial stiffness. We also determined the left ventricular mass index (LVMI), which is known to be increased in patients with diabetes [7], and which predicts cardiovascular mortality [8] in the general population.

\section{Methods}

Population We analysed baseline data from all 414 participants in the Cardiovascular Risk Factors in Patients with Diabetes - a Prospective study in Primary care (CARDIPP) who had completed the baseline study analyses by November 2008 and for whom complete data concerning nocturnal BP level and aortic PWV were available. CARDIPP is an observational prospective community-based cohort study that was launched in 2005. The general aim of the study is to explore the prevalence and impact of cardiovascular risk factors in patients with type 2 diabetes. CARDIPP provides data from an extended annual follow up of patients with type 2 diabetes, aged 5565 years, consecutively recruited by specially trained nurses from 15 different primary healthcare centres in the counties of Östergötland and Jönköping, Sweden. Patients with concomitant severe physical or mental disease (for example, terminal cancer or dementia) were not eligible for inclusion. The investigation included a standard medical history that also included enquiry about duration of diabetes and ongoing medication. Diabetes duration was defined as time from the diagnosis of type 2 diabetes until the baseline examination. The participating study centres are located in different demographic areas and differ in size, but the management and care of type 2 diabetes was organised similarly and all centres adhered to the same national guidelines for diabetes care. Among the 414 patients, there were 138 women $(33.3 \%)$. There were 111 patients $(26.8 \%)$ who were treated with diet and exercise only, 165 patients (39.9\%) who were treated with oral hypoglycaemic agents and 137 patients (33.1\%) who were treated with insulin alone or in combination with oral hypoglycaemic agents (data concerning diabetes treatment were missing for one patient). Two hundred and two patients $(48.8 \%)$ were treated with statins and 80 patients (19.3\%) were treated with acetylsalicylic acid.

Clinical evaluation Clinic-measured BP ('clinic BP') was the average of three seated measurements taken 1 min apart by specially trained nurses. Clinic normotension was defined as $\mathrm{BP}<130 / 80 \mathrm{mmHg}$ with or without blood pressure-lowering medications. Ambulatory BP measurement devices (Spacelab 90217, Spacelabs, Redmond, WA, USA) were set to measure the BP at $20 \mathrm{~min}$ intervals for $24 \mathrm{~h}$. Night-time was defined as the period between the time when the patient went to bed and the time when the patient got out of bed the following morning. Nocturnal normotension was defined as mean nighttime $\mathrm{BP}<120 / 70 \mathrm{mmHg}$ and day-time normotension was defined as mean day-time $\mathrm{BP}<135 / 85 \mathrm{mmHg}$. Applanation tonometry was performed with the SphygmoCor system (Model MM3, AtCor Medical, Sydney, NSW, Australia). From the radial pulse wave form, recorded with a Millar pressure tonometer, the corresponding ascending aortic pulse wave form was derived by the validated transfer function incorporated in the software (SphygmoCor software, version 7.0) which also calculated the central aortic BP and the central augmentation index, defined as the ratio between the late systolic peak and the early systolic peak. Aortic pulse wave transit time (measured by electrocardiogram-gated recordings of the femoral and carotid arterial pulse waves) and the surface distances between the suprasternal notch and the carotid and femoral measurement sites were used to calculate the aortic PWV. Among the 100 patients with clinical normotension, left ventricular mass was successfully determined echocardiographically [9] in 86 patients and was corrected for body surface area and expressed as LVMI.

Laboratory analysis Blood specimens were drawn in the morning following a $10 \mathrm{~h}$ overnight fast. Routine tests such as $\mathrm{HbA}_{1 \mathrm{c}}$, plasma glucose and serum lipids were analysed according to routines at the primary healthcare centres. $\mathrm{HbA}_{1 \mathrm{c}}$ values were calculated according to the Swedish Mono-S HPLC standard, which yields values approximately $1 \%$ below the DCCT standard.

Statistical analysis SPSS 16.0 (SPSS, Chicago, IL, USA) for Windows was used for statistical analyses. The statistical significance of between-group differences was tested with twosided independent $t$ tests (numerical variables), or with $\chi^{2}$ or Fisher's exact tests (nominal variables). Regression models were used to explore associations between numerical variables. Statistical significance was interpreted where $p<0.05$.

Ethics All participants gave written informed consent. The study was approved by the Regional Ethical Review Board in Linköping, Sweden and the study protocol followed the principles expressed in the Declaration of Helsinki.

\section{Results}

Masked nocturnal and masked day-time hypertension Among the 414 patients, 100 patients had clinic $\mathrm{BP}<130$ / 
$80 \mathrm{mmHg} ; 52 \%$ of these 100 participants used any blood pressure-lowering drug (beta blockers, calcium channel blockers, angiotensin converting enzyme inhibitors, angiotensin receptor blockers, or thiazide or loop diuretics). We found MNHT, defined as clinic $\mathrm{BP}<130 / 80 \mathrm{mmHg}$ and nocturnal $\mathrm{BP} \geq 120 / 70 \mathrm{mmHg}$, in 30 patients $(7.2 \%$ of the entire cohort or $30 \%$ of the clinically normotensive subgroup) and masked day-time hypertension, defined as clinic $\mathrm{BP}<130 / 80 \mathrm{mmHg}$ and day-time $\mathrm{BP} \geq 135 / 85 \mathrm{mmHg}$, in 25 patients $(6.0 \%$ of the entire cohort or $25 \%$ of the clinically normotensive subgroup). The clinical and haemodynamic characteristics of the clinically normotensive patients are presented in Table 1 according to MNHT status and in Table 2 according to masked day-time hypertension status. Compared with patients who were normotensive both clinically and at night $(n=70)$, patients with MNHT had significantly higher aortic PWV $(10.2 \pm 1.8 \mathrm{~m} / \mathrm{s}$ vs $9.4 \pm 1.7 \mathrm{~m} / \mathrm{s} ; p=0.03)$ and had higher central aortic BP $(117.6 \pm 13.9 / 74.0 \pm 9.1 \mathrm{mmHg}$ vs $110.4 \pm 16.4 / 69.7 \pm 9.6 \mathrm{mmHg} ; p=0.04)$. Compared with patients who were normotensive both clinically and during the day $(n=75)$, patients with masked day-time hypertension had similar aortic PWV and similar central aortic BP. LVMI, central pulse pressure and central augmentation index did not differ significantly according to either MNHT or masked daytime hypertension status.
Patients without antihypertensive medications Among the 414 patients, 164 patients did not use blood pressure-lowering medications; 48 of these participants had clinic $\mathrm{BP}<130$ / $80 \mathrm{mmHg}$. In this group, we found MNHT in 15 patients $(31.3 \%)$ and masked day-time hypertension in 13 patients (27.1\%). Patients with MNHT had significantly higher aortic PWV $(10.6 \pm 2.2 \mathrm{~m} / \mathrm{s}$ vs $9.0 \pm 1.5 \mathrm{~m} / \mathrm{s} ; p<0.01)$, central aortic systolic BP $(118.3 \pm 16.4 \mathrm{mmHg}$ vs $106.5 \pm 12.5 \mathrm{mmHg}$; $p<0.01)$ and central aortic diastolic BP $(74.6 \pm 7.4 \mathrm{mmHg}$ vs $68.6 \pm 8.8 \mathrm{mmHg} ; p=0.03)$ than patients who did not have MNHT. Patients with masked day-time hypertension had results for aortic PWV $(9.5 \pm 1.3 \mathrm{~m} / \mathrm{s}$ vs $9.5 \pm 2.0 \mathrm{~m} / \mathrm{s}$; $p=0.97)$, central aortic systolic BP $(114.5 \pm 15.7 \mathrm{mmHg}$ vs $108.6 \pm 14.2 \mathrm{mmHg} ; p=0.12$ ) and central aortic diastolic BP $(71.6 \pm 7.9 \mathrm{mmHg}$ vs $70.1 \pm 9.1 \mathrm{mmHg} ; p=0.22)$ similar to those of patients without masked day-time hypertension. LVMI, central pulse pressure and central augmentation index did not differ significantly according to either MNHT or masked day-time hypertension status.

Isolated nocturnal hypertension To explore the impact of isolated nocturnal hypertension, defined as clinic and day-time normotension but nocturnal hypertension, we compared all patients with clinic $\mathrm{BP}<130 / 80 \mathrm{mmHg}$ and day-time $\mathrm{BP}<135 /$ $85 \mathrm{mmHg}$ but nocturnal $\mathrm{BP} \geq 120 / 70 \mathrm{mmHg}(n=15)$ with
Table 1 Clinical and haemodynamic data in 100 clinically normotensive (clinic $\mathrm{BP}<130$ / $80 \mathrm{mmHg}$ ) patients with type 2 diabetes, with or without nocturnal hypertension (ambulatory night-time $\mathrm{BP} \geq$ $120 / 70 \mathrm{mmHg}$ )

Data missing for: ${ }^{a}$ five patients; ${ }^{\mathrm{b}}$ eight patients; ${ }^{\mathrm{c}}$ seven patients;

${ }^{\mathrm{d}}$ four patients; ${ }^{\mathrm{e}}$ three patients;

${ }^{\mathrm{f}} 16$ patients

$\mathrm{AI}$, augmentation index; DBP, diastolic blood pressure; PP, pulse pressure; SBP, systolic blood pressure

\begin{tabular}{|c|c|c|c|}
\hline Variable & Nocturnal hypertension & Nocturnal normotension & $p$ value \\
\hline$n$ & 30 & 70 & - \\
\hline Age (years) & $62.2 \pm 2.5$ & $60.6 \pm 3.1$ & 0.01 \\
\hline Women $(\%)$ & 43 & 40 & 0.83 \\
\hline Diabetes duration $^{\mathrm{a}}$ (years) & $10.5 \pm 5.9$ & $7.2 \pm 5.8$ & 0.01 \\
\hline $\mathrm{BMI}^{\mathrm{b}}\left(\mathrm{kg} / \mathrm{m}^{2}\right)$ & $29.9 \pm 6.0$ & $28.4 \pm 5.2$ & 0.23 \\
\hline $\mathrm{HbA}_{1 \mathrm{c}}(\%)$ & $6.0 \pm 1.1$ & $6.0 \pm 1.3$ & 0.84 \\
\hline Microalbuminuria $^{\mathrm{c}}(\%)$ & 7 & 3 & 0.59 \\
\hline Blood pressure-lowering medication (\%) & 50 & 53 & 0.79 \\
\hline Creatinine $^{\mathrm{d}}(\mu \mathrm{mol} / \mathrm{l})$ & $88.4 \pm 15.1$ & $88.4 \pm 13.5$ & 0.98 \\
\hline Cholesterol $^{\mathrm{e}}(\mathrm{mmol} / \mathrm{l})$ & $5.0 \pm 0.8$ & $4.8 \pm 1.2$ & 0.33 \\
\hline Clinic SBP (mmHg) & $121.8 \pm 4.9$ & $119.4 \pm 6.9$ & 0.08 \\
\hline Clinic DBP (mmHg) & $72.1 \pm 6.0$ & $70.7 \pm 6.0$ & 0.31 \\
\hline Night-time SBP (mmHg) & $125.7 \pm 8.9$ & $106.1 \pm 6.6$ & $<0.01$ \\
\hline Night-time DBP (mmHg) & $72.4 \pm 5.9$ & $61.2 \pm 4.9$ & $<0.01$ \\
\hline Day-time SBP (mmHg) & $132.5 \pm 11.1$ & $125.0 \pm 8.8$ & $<0.01$ \\
\hline Day-time DBP $(\mathrm{mmHg})$ & $78.7 \pm 6.6$ & $74.9 \pm 6.0$ & $<0.01$ \\
\hline Central SBP (mmHg) & $117.6 \pm 13.9$ & $110.4 \pm 16.4$ & 0.04 \\
\hline Central DBP (mmHg) & $74.0 \pm 9.1$ & $69.7 \pm 9.6$ & 0.04 \\
\hline Central PP (mmHg) & $43.6 \pm 11.5$ & $40.7 \pm 11.5$ & 0.25 \\
\hline Aortic PWV (m/s) & $10.2 \pm 1.8$ & $9.4 \pm 1.7$ & 0.03 \\
\hline $\operatorname{LVMI}^{\mathrm{f}}\left(\mathrm{g} / \mathrm{m}^{2}\right)$ & $118.9 \pm 22.3$ & $118.6 \pm 30.5$ & 0.97 \\
\hline AI $(\%)$ & $26.1 \pm 7.3$ & $27.1 \pm 8.9$ & 0.59 \\
\hline
\end{tabular}


Table 2 Clinical and haemodynamic data in 100 clinically normotensive (clinic $\mathrm{BP}<130$ / $80 \mathrm{mmHg}$ ) patients with type 2 diabetes, with or without day-time hypertension (ambulatory day-time $\mathrm{BP} \geq 135$ / $85 \mathrm{mmHg}$ )

Data missing for: ${ }^{a}$ five patients; ${ }^{\mathrm{b}}$ eight patients; ${ }^{\mathrm{c}}$ seven patients;

${ }^{\mathrm{d}}$ four patients; ${ }^{\mathrm{e}}$ three patients;

${ }^{\mathrm{f}} 16$ patients

AI, augmentation index; DBP, diastolic blood pressure; PP, pulse pressure; SBP, systolic blood pressure

\begin{tabular}{|c|c|c|c|}
\hline Variable & Day-time hypertension & Day-time normotension & $p$ value \\
\hline$n$ & 25 & 75 & - \\
\hline Age (years) & $61.8 \pm 3.0$ & $60.9 \pm 3.0$ & 0.17 \\
\hline Women $(\%)$ & 48 & 61 & 0.41 \\
\hline Diabetes duration $^{\mathrm{a}}$ (years) & $9.5 \pm 6.5$ & $7.7 \pm 5.8$ & 0.20 \\
\hline $\mathrm{BMI}^{\mathrm{b}}\left(\mathrm{kg} / \mathrm{m}^{2}\right)$ & $29.0 \pm 6.8$ & $28.7 \pm 5.0$ & 0.81 \\
\hline $\mathrm{HbA}_{1 \mathrm{c}}(\%)$ & $5.8 \pm 0.9$ & $6.1 \pm 1.3$ & 0.34 \\
\hline Microalbuminuria $^{\mathrm{c}}(\%)$ & 4 & 4 & 1.00 \\
\hline Blood pressure-lowering medication (\%) & 50 & 53 & 0.64 \\
\hline Creatinine $^{\mathrm{d}}(\mu \mathrm{mol} / \mathrm{l})$ & $85.6 \pm 14.4$ & $89.3 \pm 13.7$ & 0.27 \\
\hline Cholesterol $^{\mathrm{e}}(\mathrm{mmol} / \mathrm{l})$ & $4.9 \pm 0.9$ & $4.9 \pm 1.2$ & 0.99 \\
\hline Clinic SBP (mmHg) & $122.5 \pm 4.7$ & $119.3 \pm 6.7$ & 0.03 \\
\hline Clinic DBP (mmHg) & $71.3 \pm 4.9$ & $71.1 \pm 6.3$ & 0.88 \\
\hline Night-time SBP (mmHg) & $122.4 \pm 11.4$ & $108.5 \pm 9.5$ & $<0.01$ \\
\hline Night-time DBP (mmHg) & $68.8 \pm 8.1$ & $63.2 \pm 6.5$ & $<0.01$ \\
\hline Day-time SBP (mmHg) & $140.1 \pm 6.5$ & $123.0 \pm 7.0$ & $<0.01$ \\
\hline Day-time DBP (mmHg) & $81.0 \pm 6.4$ & $74.4 \pm 5.5$ & $<0.01$ \\
\hline Central SBP (mmHg) & $116.6 \pm 15.0$ & $111.2 \pm 16.2$ & 0.15 \\
\hline Central DBP $(\mathrm{mmHg})$ & $71.9 \pm 9.4$ & $70.7 \pm 9.7$ & 0.57 \\
\hline Central PP (mmHg) & $44.6 \pm 11.9$ & $40.5 \pm 11.3$ & 0.12 \\
\hline Aortic PWV (m/s) & $10.0 \pm 1.3$ & $9.5 \pm 1.9$ & 0.19 \\
\hline LVMI $\left(\mathrm{g} / \mathrm{m}^{2}\right)$ & $119.8 \pm 19.0$ & $118.3 \pm 31.0$ & 0.80 \\
\hline $\mathrm{AI}(\%)$ & $27.5 \pm 7.8$ & $26.5 \pm 8.7$ & 0.62 \\
\hline
\end{tabular}

patients who were normotensive clinically as well as during both the day and the night $(n=60)$. Central aortic diastolic BP was significantly higher in patients with isolated nocturnal hypertension $(75.9 \pm 8.5 \mathrm{mmHg}$ vs $69.4 \pm 9.6 \mathrm{mmHg}$; $p=0.02$ ). Central aortic systolic BP and aortic PWV were higher in patients with isolated nocturnal hypertension than in patients who were normotensive according to all three measurements, but the differences were not statistically significant (central aortic systolic BP: $116.6 \pm 13.9 \mathrm{mmHg}$ vs $109.8 \pm 16.5 \mathrm{mmHg} ; p=0.15$ and aortic PWV $10.3 \pm 2.4$ vs $9.3 \pm 1.7 ; p=0.06)$. LVMI, central pulse pressure and central augmentation index did not differ significantly according to isolated nocturnal hypertension status.

Predictors of nocturnal systolic blood pressure We investigated the independent determinants of nocturnal systolic $\mathrm{BP}$ in all patients with clinical normotension $(n=100)$, using a multivariable regression analysis in which nocturnal systolic BP was the dependent variable and in which age, sex, BMI, blood pressure-lowering medication, $\mathrm{HbA}_{1 \mathrm{c}}$ and aortic PWV were entered as independent variables. Only aortic PWV predicted nocturnal systolic BP significantly and independently $(r=0.23 ; p=0.05)$. This association, however, did not remain statistically significant $(r=0.20$; $p=0.09$ ) following the addition of creatinine as an additional independent variable.
Predictors of arterial stiffness To identify the variables that were independently associated with arterial stiffness, we performed a multivariable regression analysis in all patients with clinical normotension $(n=100)$. Aortic PWV was the dependent variable and age, sex, BMI, blood pressurelowering medication, $\mathrm{HbA}_{1 \mathrm{c}}$, cholesterol, creatinine, diabetes duration and $24 \mathrm{~h}$ ambulatory mean arterial pressure were entered as independent variables. As seen in Table 3,

Table 3 Determinants of aortic pulse wave velocity in a multivariable regression analysis performed in 100 normotensive (clinic $\mathrm{BP}<130$ / $80 \mathrm{mmHg}$ ) patients with type 2 diabetes

\begin{tabular}{|c|c|c|}
\hline Independent variable & $r$ & $p$ value \\
\hline Age (years) & 0.11 & 0.34 \\
\hline Sex & -0.04 & 0.77 \\
\hline BMI $\left(\mathrm{kg} / \mathrm{m}^{2}\right)$ & 0.23 & 0.05 \\
\hline Blood pressure-lowering medication (yes/no) & 0.13 & 0.29 \\
\hline $\mathrm{HbA}_{1 \mathrm{c}}(\%)$ & 0.25 & 0.03 \\
\hline Cholesterol (mmol/l) & 0.09 & 0.43 \\
\hline Creatinine $(\mu \mathrm{mol} / \mathrm{l})$ & 0.06 & 0.68 \\
\hline Diabetes duration (years) & 0.19 & 0.09 \\
\hline Ambulatory MAP (mmHg) & 0.15 & 0.20 \\
\hline
\end{tabular}

Model's $r$ square 0.20

MAP, Mean arterial pressure 
aortic PWV was significantly associated with only $\mathrm{HbA}_{1 \mathrm{c}}$ $(r=0.25 ; p=0.03)$ and BMI $(r=0.23 ; p=0.05)$.

\section{Discussion}

In our cohort of middle-aged patients with type 2 diabetes, approximately one out of three patients with clinic $\mathrm{BP}<130$ / $80 \mathrm{mmHg}$ had night-time BP values $\geq 120 / 70 \mathrm{mmHg}$. Patients with normal clinic BP but nocturnal hypertension had higher aortic PWV and higher central BP than patients with normal BP both in the clinic and at night. As the risk for cardiovascular morbidity and mortality increases with both aortic stiffness [5] and central BP [6], it is likely that patients with MNHT have higher cardiovascular risk than patients with normotension both in the clinic and at night. This suggests that clinical BP measurements may not provide sufficient information in middle-aged patients with type 2 diabetes. Indeed, it was recently shown that in Japanese patients with type 2 diabetes, both night- and day-time systolic BP predicted cardiovascular events independently of clinic systolic BP levels [10]. Our findings offer a plausible mechanism to explain the higher risk attributable to a higher ambulatory BP.

In the general population, night-time $\mathrm{BP}$ is a better predictor of fatal cardiovascular events than day-time BP [11] and the risk for cardiovascular death increases more steeply with increasing night-time BP levels than with increasing day-time BP levels [12]. Despite this, in previous investigations of the prevalence of masked hypertension in diabetes, the definition of masked hypertension has been restricted to out-of-clinic BP values obtained during the awake part of the day, using either self-measured BP [1, 2] or ambulatory BP [3, 4, 13]. We report for the first time the prevalence of MNHT in type 2 diabetes, and conclude that it is more common than masked day-time hypertension and identifies more accurately the patients at elevated risk for macrovascular complications of diabetes. As in several previous investigations in patients with [2] or without [14-16] diabetes, we included patients with masked uncontrolled hypertension (i.e. patients on blood pressure-lowering drug therapy who reach the BP goal according to clinic but not ambulatory measurements). In contrast to previous reports of masked hypertension in diabetes, however, we defined normal clinic BP according to current diabetes guidelines, where $130 / 80 \mathrm{mmHg}$ constitutes both the diagnostic threshold and the treatment target for hypertension [17]. The current guidelines provide no diabetes-specific ambulatory hypertension thresholds, however, and we used 120/70 $\mathrm{mmHg}$ as the nocturnal BP cut-off value and 135/85 $\mathrm{mmHg}$ as day-time cut-off value, as proposed for patients with hypertension [18]. The same cut-off values for nocturnal and day-time systolic BP have been associated with a good prognosis in patients with type 2 diabetes [10].

It has been suggested that in patients with mild renal dysfunction, the reduced day-time renal sodium excretion is compensated by elevated nocturnal BP levels that enhance pressure natriuresis in the night [19]. It was also recently shown that in patients with reduced renal function, BP remains high for a longer period of time after going to bed compared with patients with normal renal function [20]. Previous investigators have also found that a blunted diurnal BP pattern is associated with the prevalence of nephropathy in patients with type 2 diabetes [21]. In the light of these findings, we find it noteworthy that in our cohort of clinically normotensive patients, nocturnal systolic BP was independently associated with aortic PWV after adjustments for age, sex, BMI, $\mathrm{HbA}_{1 \mathrm{c}}$ and blood pressure-lowering medication, but that this association did not remain significant after further adjustments for creatinine levels. Although we cannot distinguish between causes and consequences in our observational study, our findings lend support to a renal-dependent association between nocturnal systolic BP levels and arterial stiffness in patients with type 2 diabetes.

Central augmentation index is a surrogate marker for arterial stiffness. It is influenced by several factors such as the strength of the ventricular ejection, the heart rate, the aortic PWV itself and the site of peripheral arterial pulse wave reflection, and thus provides only indirect information on arterial stiffness [22]. We found no significant difference in central augmentation index between patients with or without MNHT, although aortic PWV did differ. This finding is consistent with the previous observation that there is no association between increased aortic PWV and central augmentation index elevation in patients with diabetes [23]. The recent and noteworthy observation that increased central aortic impedance may occur before elevation of aortic PWV in patients with type 1 diabetes [24] remains to be formally tested in patients with type 2 diabetes. We have not performed such an analysis in our study and thus cannot report any data concerning central aortic impedance and its possible relationship to MNHT.

Some of our findings are contradictory to those previously observed. First, it has been shown that in women with type 1 diabetes, aortic stiffness is positively associated with diabetes duration [25] but in our cohort we found no association between diabetes duration and aortic PWV following adjustment for traditional risk factors. However, because of the slower onset of hyperglycaemic symptoms in patients with type 2 diabetes compared with type 1 , some patients in our cohort may have been hyperglycaemic for a considerable period of time before diabetes was diagnosed and this may have obscured a 
correlation between diabetes duration and aortic stiffness. Second, although central blood pressure is a known predictor of left ventricular mass in patients with type 2 diabetes [26], we found no such associations in our cohort. This may reflect the relatively high use of BP-lowering medications in our cohort.

Certain limitations of our study should be recognised. First, MNHT status was determined according to a single ambulatory BP measurement. The high degree of intra-individual variation of nocturnal BP patterns in patients with hypertension [27] means that it could be argued that repeated ambulatory BP measurements are needed in order to determine the true prevalence of MNHT. However, it was recently shown that short-term reproducibility of nocturnal BP patterns is higher in patients with type 2 diabetes (88.9\%) than in patients without diabetes (70.5\%) [28]. This suggests that nocturnal BP patterns may be accurately determined by a single ambulatory measurement in the majority of patients with type 2 diabetes. Furthermore, we defined night-time as the time patients actually reported spending in bed, as opposed to using an arbitrary definition of night-time, as the latter approach has previously been shown to lead to misclassification of diurnal BP patterns [29] and also the main changes in BP between night and day are not dependent on the sleep per se, but on ambulation [30].

Second, administration of blood pressure-lowering medications at different times of the day may have influenced the diurnal BP pattern. We do not have information on the timing of the blood pressure-lowering medications. However, in a large Spanish cohort of treated hypertensive patients, the investigators found no association between the time of the day at which blood pressure-lowering medications were administered and the circadian BP patterns [31]. Furthermore, we believe that the consistency of our main findings in the subgroup of patients who did not use blood pressure-lowering medications shows that the associations we report are not heavily influenced by the intake of blood pressure-lowering drugs.

Third, obstructive sleep apnoea syndrome may underlie some of the cases of MNHT, and may have contributed to some extent to the increased risk profile seen in these patients, but we have no data concerning its prevalence in our cohort. However, we have no reason to believe that the prevalence of obstructive sleep apnoea syndrome is different in our cohort than in other populations of middle-aged patients with type 2 diabetes and with a similar prevalence of obesity.

Fourth, sleep depth has been shown to influence nocturnal BP levels, but was not measured in our study. Importantly, though, although the ambulatory BP measurement device itself has been shown to cause arousals, nocturnal BP has been shown not to be influenced by these measurement-induced arousals [32]. Thus, although the sleep quality of the participants in the present study was not evaluated and may have been impaired by the ambulatory BP-measuring device, we do not believe that this influenced the estimated prevalence of MNHT.

We conclude that nocturnal hypertension is commonly found in patients with type 2 diabetes and clinic BP levels below the currently recommended diagnostic threshold and treatment goal. Identification of patients with MNHT has the potential to improve risk stratification as these patients had increased arterial stiffness and higher central BP. Therefore, ambulatory BP evaluation is suggested in patients with type 2 diabetes and BP levels $<130 / 80 \mathrm{mmHg}$. For optimal identification of patients with elevated risk, patients with MNHT rather than masked day-time hypertension should be sought. Further studies are needed to clarify the long-term prognostic significance of MNHT in type 2 diabetes, and to determine whether intensified medical treatment of these patients would be beneficial. Outcome-driven diabetes-specific thresholds for optimal ambulatory BP levels are urgently needed in order to improve the quality of care of patients with diabetes.

Acknowledgements This study was supported by grants from the Medical Research Council of Southeast Sweden, the Center for Medical Image Science and Visualization, the Faculty of Linköping University, Futurum, GE Healthcare, the Swedish Heart-Lung Foundation, Medical Research Council Grant 12661 and the Diabetes Research Centre.

Duality of interest The authors declare that there is no duality of interest associated with this manuscript.

\section{References}

1. Kamoi K, Miyakoshi M, Soda S, Kaneko S, Nakagawa O (2002) Usefulness of home blood pressure measurement in the morning in type 2 diabetic patients. Diabetes Care 25:2218-2223

2. Sakaguchi K, Horimatsu T, Kishi M et al (2005) Isolated home hypertension in the morning is associated with target organ damage in patients with type 2 diabetes. J Atheroscler Thromb $12: 225-231$

3. Marchesi C, Maresca AM, Solbiati F et al (2007) Masked hypertension in type 2 diabetes mellitus. Relationship with leftventricular structure and function. Am J Hypertens 20:1079-1084

4. Leitão CB, Canani LH, Kramer CK, Boza JC, Pinotti AF, Gross JL (2007) Masked hypertension, urinary albumin excretion rate, and echocardiographic parameters in putatively normotensive type 2 diabetic patients. Diabetes Care 30:1255-1260

5. Cruickshank K, Riste L, Anderson SG, Wright JS, Dunn G, Gosling RG (2002) Aortic pulse wave velocity and its relationship to mortality in diabetes and glucose intolerance: an integrated index of vascular function? Circulation 106:2085-2090

6. Roman MJ, Devereux RB, Kizer JR et al (2007) Central pressure more strongly relates to vascular disease and outcome than does brachial pressure: the Strong Heart Study. Hypertension 50:197203

7. Devereux RB, Roman MJ, Paranicas M et al (2000) Impact of diabetes on cardiac structure and function: the strong heart study. Circulation 101:2271-2276 
8. Levy D, Garrison RJ, Savage DD, Kannel WB, Castelli WP (1990) Prognostic implications of echocardiographically determined left ventricular mass in the Framingham heart study. N Engl J Med 322:1561-1566

9. Devereux RB, Reichek N (1977) Echocardiographic determination of left ventricular mass in man. Anatomic validation of the method. Circulation 55:613-618

10. Eguchi K, Pickering TG, Hoshide S et al (2008) Ambulatory blood pressure is a better marker than clinic blood pressure in predicting cardiovascular events in patients with/without type 2 diabetes. Am J Hypertens 21:443-450

11. Boggia J, Li Y, Thijs L et al (2007) Prognostic accuracy of day vs night ambulatory blood pressure: a cohort study. Lancet 370:1219-1229

12. Dolan E, Stanton A, Thijs L et al (2005) Superiority of ambulatory over clinic blood pressure measurement in predicting mortality: the Dublin outcome study. Hypertension 46:156161

13. Strachan MW, Gough K, McKnight JA, Padfield PL (2002) Ambulatory blood pressure monitoring: is it necessary for the routine assessment of hypertension in people with diabetes? Diabet Med 19:787-789

14. Hara A, Ohkubo T, Kikuya M et al (2007) Detection of carotid atherosclerosis in individuals with masked hypertension and white-coat hypertension by self-measured blood pressure at home: the Ohasama study. J Hypertens 25:321-327

15. Bobrie G, Chatellier G, Genes N et al (2004) Cardiovascular prognosis of "masked hypertension" detected by blood pressure self-measurement in elderly treated hypertensive patients. JAMA 291:1342-1349

16. Ohkubo T, Kikuya M, Metoki H et al (2005) Prognosis of "masked" hypertension and "white-coat" hypertension detected by 24-h ambulatory blood pressure monitoring 10-year follow-up from the Ohasama study. J Am Coll Cardiol 46:508-515

17. American Diabetes Association (2008) Standards of medical care in diabetes 2008. Diabetes Care 31(Suppl 1):S12-S54

18. Mancia G, De Backer G, Dominiczak A et al (2007) 2007 Guidelines for the management of arterial hypertension: The Task Force for the Management of Arterial Hypertension of the European Society of Hypertension (ESH) and of the European Society of Cardiology (ESC). J Hypertens 25:1105-1187

19. Fukuda M, Goto N, Kimura G (2006) Hypothesis on renal mechanism of non-dipper pattern of circadian blood pressure rhythm. Med Hypotheses 67:802-806
20. Fukuda M, Mizuno M, Yamanaka T et al (2008) Patients with renal dysfunction require a longer duration until blood pressure dips during the night. Hypertension 52:1155-1160

21. Knudsen ST, Poulsen PL, Hansen KW, Ebbehøj BT, Mogensen CE (2002) Pulse pressure and diurnal blood pressure variation: association with micro- and macrovascular complications in type 2 diabetes. Am J Hypertens 15:244-250

22. Laurent S, Cockcroft J, Van Bortel L et al (2006) Expert consensus document on arterial stiffness: methodological issues and clinical implications. Eur Heart J 27:2588-2605

23. Lacy PS, O'Brien DG, Stanley AG, Dewar MM, Swales PR, Williams B (2004) Increased pulse wave velocity is not associated with elevated augmentation index in patients with diabetes. J Hypertens 22:1937-1944

24. Sweitzer NK, Shenoy M, Stein JH et al (2007) Increases in central aortic impedance precede alterations in arterial stiffness measures in type 1 diabetes. Diabetes Care 30:2886-2891

25. Ahlgren AR, Sundkvist G, Wollmer P, Sonesson B, Länne T (1999) Increased aortic stiffness in women with type 1 diabetes mellitus is associated with diabetes duration and autonomic nerve function. Diabet Med 4:291-297

26. Sharman JE, Fang ZY, Haluska B, Stowasser M, Prins JB, Marwick TH (2005) Left ventricular mass in patients with type 2 diabetes is independently associated with central but not peripheral pulse pressure. Diabetes Care 28:937-939

27. Cuspidi C, Meani S, Salerno M et al (2004) Reproducibility of nocturnal blood pressure fall in early phases of untreated essential hypertension: a prospective observational study. J Hum Hypertens 18:503-509

28. Cuspidi C, Meani S, Lonati L et al (2006) Short-term reproducibility of a non-dipping pattern in type 2 diabetic hypertensive patients. J Hypertens 24:647-653

29. Nyström F, Malmström O, Karlberg BE, Öhman KP (1996) Twenty-four hour ambulatory blood pressure in the population. $\mathrm{J}$ Intern Med 240:279-284

30. Khoury AF, Sunderajan P, Kaplan NM (1992) The early morning rise in blood pressure is related mainly to ambulation. Am J Hypertens 5:339-344

31. de la Sierra A, Redon J, Banegas JR et al (2009) Prevalence and factors associated with circadian blood pressure patterns in hypertensive patients. Hypertension 53:466-472

32. Schwan Å, Eriksson G (1992) Effect on sleep-but not on blood pressure - of nocturnal non-invasive blood pressure monitoring. J Hypertens 10:189-194 